\title{
OUTPATIENTS APPOINTMENT SCHEDULING WITH MULTI-DOCTOR SHARING RESOURCES
}

\author{
Nara Yeon \\ Taesik Lee \\ Hoon Jang \\ Korea Advanced Institute of \\ Science and Technology \\ 335 Gwahangno, Yuseong-gu \\ Daejeon, 305-701, REPUBLIC OF KOREA
}

\begin{abstract}
In an outpatient department of general hospitals, several doctors practice simultaneously. While individual doctors have their own patient panel and work independently, they share common resources such as space, personnel and equipments. In such settings, designing an optimal scheme to manage patient flow, e.g. appointment scheduling, requires to consider patient flows for all doctors instead of focusing on a single doctor. This paper examines an appointment scheduling problem for an outpatient unit where multiple doctors practice independently yet sharing common resources. An ophthalmology department of a large-scale general hospital in Korea is modeled in discrete event simulation. Our experimental results show that under multiple-doctor and resource-sharing environment, collection of the seemingly optimal appointment rules for individual doctors does not lead to optimal performance for the system. It implies that altering a patient flow, especially modifying the scheduling rule, should consider the interdependence effects within the system.
\end{abstract}

\section{INTRODUCTION}

Outpatient units - outpatient clinics or outpatient departments in a large hospital - carry a significant load in a healthcare delivery system. It is generally an entry point to a healthcare system for non-urgent patients, and a major channel for delivering cure. For example, in the hospital where the authors conducted a patient flow case study, there are about 4,000 outpatients per day versus 1,000 inpatients and a relatively small number of other types such as ER patients.

Effectively managing patient flow in an outpatient unit is a key to achieving operational excellence as well as ensuring clinical quality. It is especially so for an outpatient department in a large hospital as it handles very large volume of patients with a diverse case mix. Symptoms of suboptimal patient flow in an outpatient unit include long waiting time, excessive appointment backlog, and overtime by staff, all of which result in dissatisfaction for external and internal customers, and even potential harm to patients.

Patient flow management requires addressing three aspects of an outpatient unit: arrival of patients, service process, and queuing process. Working on the patient's arrival includes controlling its patient panel size, balancing patient volumes across available sessions, and achieving desirable patient arrival pattern within a session. The goal here is to improve alignment between the unit's service capacity and demand. Another approach is to improve the unit's service process. By redesigning its work flow and enhancing communication, the effective capacity can be increased to positively affect the patient flow. Alternatively, queuing process can be altered to minimize adverse consequences of waiting within the unit. 
We may seek a better prioritization of waiting patients, reduce the time when a patient has to be present physically in the waiting room, and implement value-adding activities for patients during their waiting time.

As many outpatient units run on an appointment-basis, appointment scheduling is one of the fundamental design parameters for managing patient flows. Appointment scheduling is defined as, for a given outpatient session, determining the number of patients to assign into a given time interval, called a slot, and the beginning time for each slot. Appointment scheduling is a basic tool to control a temporal distribution of arriving patients to achieve the most desirable work load patterns in an outpatient unit.

Many prior researches have been conducted on outpatient scheduling. According to a comprehensive review on the previous work on outpatient scheduling by Cayirli et al. (2003), the earliest study is a work by Bailey (1952) who suggested 2BEG rule. 2BEG rule sets two patients at the first slot of a given session, and then evenly distributes patients into the rest of the session by the average service time. Blanco et al. (1964) studied appointment rules in the presence of uncertainty in patient arrival, and showed that multiple block appointment rules perform better than single block appointment rules. Cayirli et al. (2006) (2008) tested several appointment rules under various operational environments to examine whether patient's sequence scheme is effective or not. They found that 2BEG, IBFI and MBFI rules are generally superior to other rules.

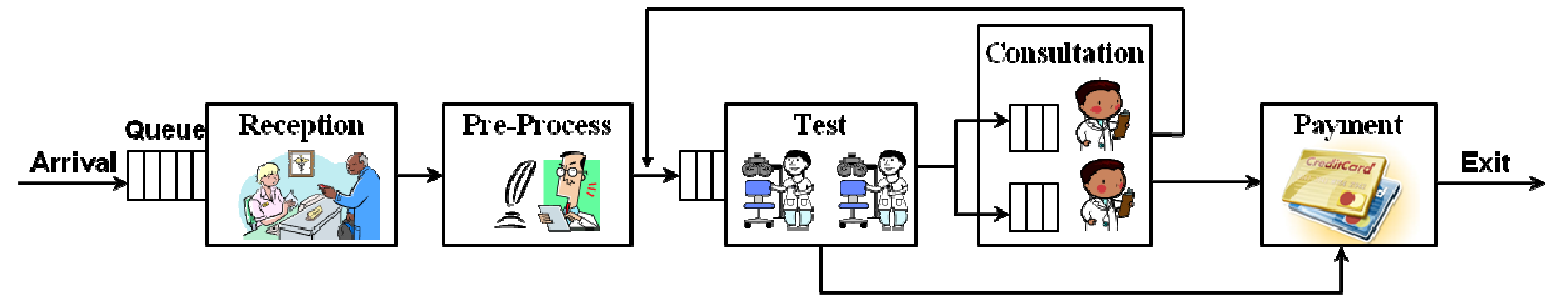

Figure 1: The flowchart shows general process an outpatient goes through in the outpatient department. Consultation and Test can be occurred several times a visit. A patient only sees the designated doctor but share common resources at 'Reception' and 'Test'. Especially at 'Test', medical equipments and test rooms and staffs are shared among the patients from different specialists.

Most of those studies have focused on a single-doctor environment. In practice, however, single doctor cases are hardly pragmatic because it is typical to have multiple doctors practicing simultaneously in an outpatient unit. When these doctors affect the patient flows of each others' by sharing some part of the unit's resources, as depicted in figure 1, the decisions by each doctor to change its patient flows affect not only his/her patient flow, but also other doctors'. As such, best rules previously identified for a singledoctor environment may not be effective in improving the performance of an overall system.

This paper examines outpatient appointment scheduling where multiple doctors practice individually, yet sharing common resources between them. Our aim is to assert that appointment scheduling must be derived as a system problem in order to achieve a system-wise performance improvement.

\section{STUDY DESIGN AND METHODS}

Whether it is a single-doctor clinic or an outpatient department where multiple doctors practice together, each doctor serves his/her own patient panels. To be more specific, a doctor has control over his/her patients only to decide on their appointment schedule and process flow, not other doctors' patients. Thus, a doctor is likely to determine a scheduling rule with only his/her outpatient patients considered. In other words, a typical appointment scheduling practice for a multiple-doctor outpatient unit can be simply viewed as a collection of several independent single-doctor problems.

It is reasonable to expect, however, that appointment schedules for multiple doctors must be determined as a collective set rather than a mere collection of individually-made decisions. So in this study, we compare patient flow performances of multiple-doctor, resource-sharing outpatient unit under two ap- 
pointment rule design scenarios: 1) an appointment rule for each doctor is determined by individual doctor's preference, and 2) a collective set, simultaneously considering patient flows by all doctors, is examined to determine an optimal set of appointment rules.

Three appointment scheduling rules have been selected from the literature as a set of candidate rules each doctor can take from. They were developed for a single-doctor case in several prior researches. An ophthalmology department in a large hospital was chosen to model a multiple-doctor, resource-sharing environment. A discrete event simulation model is created for the ophthalmology outpatient department to conduct virtual experiments. Various combinations of the three rules are applied to the model for scheduling patient appointments for individual doctors, and their patient flow performance is evaluated. Following sections describe the details of each component of our study methods.

\subsection{Performance Measure}

For appointment scheduling problems, two mostly considered performance measures are patient's waiting time $(W)$ and staff's over time $(O)$. This study also adopts the two measures in evaluating the effectiveness of appointment schedules.

Waiting time, $W$ is defined for a given session as the average of total waiting time (minute) experienced by individual patients during their stay in an outpatient unit:

$$
W=\frac{\sum_{i=1}^{n} W_{i}}{n}
$$

where $n$ is the total number of patients in a session, and $W_{i}$ is the waiting time experienced by an individual patient $i$.

Overtime is defined for care providers to measure the amount of work they have to do beyond their regular working hours. Considering doctors are the most valuable assets in a typical outpatient unit, we define overtime, $O$, to be the average overtime (minute) experienced by all doctors in a session:

$$
O=\frac{\sum_{k=1}^{D} O_{k}}{D}
$$

$O_{k}$ is overtime experienced by a doctor $k$, and $D$ is the number of doctors working in a given session. As a final performance measure, a cost function, $f$ is defined as a weighted sum of $W$ and $O$ :

$$
f=C_{W} W+C_{O} O
$$

$C_{w}$ and $C_{O}$ are coefficients to represent a relative cost associated with patient's waiting time and doctor's overtime respectively. For simpler analysis, $C_{w}$ and $C_{O}$ have a value between 0 and 1 , and their summation is equal to 1 . Different organizations have different value structure for these costs, and thus it is difficult to assign a universal value for these coefficients. On the other hand, for the same system, different values for these coefficients change the best appointment scheduling solutions for the system. (Ho and Lau, 1992) Thus, we use an efficient frontier analysis to deal with changes in coefficient sets.

\subsection{Appointment Rules}

For this study, we selected three scheduling rules - 2BEG, IBFI, and MBFI - that have been suggested in Cayirli et al. (2006, 2008) as generally superior appointment scheduling rules. These rules are used in our experiments as a possible appointment scheduling solution for each doctor in a session. Table 1 shows brief descriptions of the selected rules. 
Yeon, Lee and Jang

Table 1: Three candidate scheduling rules for a single doctor case (adapted from Cayirli et al. (2006))

\begin{tabular}{c|l|l|l|l|}
\hline Rules & \multicolumn{1}{|c}{ Description } & \multicolumn{1}{|c}{ Visualization } \\
\hline $\begin{array}{c}\text { 2BEG } \\
\text { (Bailey,1952) }\end{array}$ & $\begin{array}{l}\text { Assign two patients at the beginning of the session and distri- } \\
\text { bute remaining patients based on their average service time } \\
\text { IBFI }\end{array}$ & $\begin{array}{l}\text { Evenly distribute all patients based on their average service time } \\
\text { Assign two patients to a time interval whose length is twice the } \\
\text { average service time of a patient }\end{array}$ & & \\
\hline
\end{tabular}

\subsection{Experiment Settings}

Experiments to compare the effectiveness of a set of appointment rules are carried out for an ophthalmology department of a large general hospital in Korea. The department operates two sessions per day, a morning session and an afternoon session, from Monday to Friday. We selected two most crowded sessions of the department: Wednesday afternoon and Friday morning. Wednesday PM session hosts three specialists $\left(\mathrm{S}_{\mathrm{W} 1}, \mathrm{~S}_{\mathrm{W} 2}, \mathrm{~S}_{\mathrm{W} 3}\right)$. For Friday $\mathrm{AM}$ session, four specialists, $\left(\mathrm{S}_{\mathrm{F} 1}, \mathrm{~S}_{\mathrm{F} 2}, \mathrm{~S}_{\mathrm{F} 3}, \mathrm{~S}_{\mathrm{F} 4}\right)$ practice simultaneously.

Our experiments compare the performance of solutions obtained from two approaches. In the first approach, an appointment scheduling rule for each doctor is determined by considering each doctor's patient flow separately. The three candidate rules are evaluated for one doctor's, say $S_{\mathrm{W}_{1}}$, patient flow performance, and a rule with the best performance is selected for the doctor. Since the performance of each candidate rule is dependent on the other doctors' appointment rules, we assume that an appointment scheduling rule with the minimum expected waiting time and overtime under various conditions is selected. A collection of these best rules for individual doctors gives collective appointment scheduling rules for the session. In the second approach, we presume that the interdependence among patient flows by the doctors is significant, and appointment rules for the simultaneously practicing doctors are considered together as a set. We predict that a collection of individually chosen rules may not yield the overall best results, and thus performance measure for the whole unit is considered to select the overall best set of appointment rules.

The best set of appointment scheduling rules can be determined by conducting full factorial experiments. For Wednesday AM session, with the three doctors and three candidate rules, a full factorial experiment would require 27 experiments. For Friday PM session, it is 81 experiments. As each experiment is done with a relatively simple simulation, a full factorial experiment is certainly possible in our study, even with a large number of simulation replication runs. Yet, as the number of doctors working in a session increases and the number of candidate rules gets large, a full factorial experiment would become less feasible. Therefore, for the sake of generality, we present an experimental framework based on orthogonal arrays. Orthogonal arrays for Wednesday PM session and Friday AM session are shown in Appendix B.

\section{BUILDING A SIMULATION MODEL OF AN OUTPATIENT DEPARTMENT}

\subsection{Ophthalmology Department Under Consideration}

Our reference model is an outpatient department of ophthalmology in a general hospital in Korea. It runs two sessions a day, and for each session, multiple doctors practice simultaneously. Due to the nature of ophthalmology, arriving patients go through a few processes before seeing their doctor. These processes include a vision test, measuring intraocular pressure, preliminary consultations, and many others. Some of 


\section{Yeon, Lee and Jang}

these are required for all patients, and some are required for a certain set of patients. Once a patient is done with these processes, the patient meets with his/her doctor. After the consultation (or treatment), the patient may leave the department or go to the exam rooms in the department to get some tests done as instructed by the doctor. It is these pre- and post-consultation processes that patients for each doctor share common resource of the department such as test equipments and nursing staff.

The outpatient department operates mostly on an appointment basis, but there is a small fraction of patients who arrive randomly. These are the patients referred from other departments such as Emergency Department in the hospital. They arrive randomly without an appointment, and take up 5 10\% of total outpatient volume. These randomly arriving patients are seen by supporting residents, not by specialists.

\subsection{Data collection and Data preparation}

Data needed to build a simulation model have been obtained through multiple sources. First, we collected time data available in the department's EMR (Electronic Medical Record) system. EMR data from October 1 to December 31, 2007, totaling more than 15000 patient-visits, was collected. Data that are not available from the EMR were estimated based on the direct observations and interviews of doctors and nurses of the department. For example, the data for exact arrival times of walk-in patients from other department was unavailable, and only the rough estimation of the average number of those patients was available. With limited information, we assumed exponential distribution to model inter-arrival time of the walk-in patients, referring to Swarzman (1970). Their arrival rate is approximated from the average number of walk-in patients in a session. Table 2 summarizes input data used to build a simulation model.

Table 2: The input data for the simulation model. Specific values are shown in Appendix A.

\begin{tabular}{|c|c|}
\hline Input data & Description \\
\hline Service Time Distribution & $\begin{array}{l}\text { - Doctor's consultation time } \\
\text { - Medical examination time for various types } \\
\text { - Others(billing, registration, etc) }\end{array}$ \\
\hline Walk-in Patients Distribution & $\begin{array}{l}\text { - Walk-in patient arrival rate } \\
\text { - Exam request rate }\end{array}$ \\
\hline Punctuality of Patient Arrival & - Punctuality of the patients with respect to their appointment time \\
\hline No-show Rate & - No-show rate of the patients with appointment \\
\hline New-patient Ratio & - Fraction of new visits out of total visits \\
\hline Process Pattern Ratio & $\begin{array}{l}\text { - The Ratio of patient process flow types for the five flows } \\
\text { - Consultation } \\
\text { - Consultation - Examination } \\
\text { - Consultation - Examination - Consultation } \\
\text { - Examination } \\
\text { - Examination - Consultation }\end{array}$ \\
\hline $\begin{array}{l}\text { Post-consultation Examination } \\
\text { Ratio }\end{array}$ & $\begin{array}{l}\text { - Ratio of doctor } i \text { 's patients to whom doctor } i \text { prescribes a specific } \\
\text { medical exam after the consultation }\end{array}$ \\
\hline Resource Capacity & $\begin{array}{l}\text { - Number of medical equipments } \\
\text { - Number of related personnel } \\
\text { - Available medical exams in each medical exam room }\end{array}$ \\
\hline
\end{tabular}




\subsection{Simulation Model}

A discrete event simulation model was constructed using ARENA ${ }^{\mathrm{TM}}$. To a large degree, the simulation model is an exact replication of the real environment of the department.

Specialists, denoted as $S_{\mathrm{W} 1}$ for example, have their own patient panels. A process flow that an individual patient follows is determined by the predefined probability for each doctor -Process Pattern Ratio, New-patient Ratio and Post-consultation Examination Ratio. There are two types of patients assigned to each specialist; patients with a scheduled appointment and the patients for post-surgery consultation. Patients with a scheduled appointment are the major interests in this study as their arrival pattern is controlled by the appointment rules in question. Volume of these patients takes up almost $90 \%$ of the total outpatients of the department. Patients of post-surgery consultation come see their doctor after a surgery to get a post-surgery consultation and some follow-up exams in the department. These patients do not make an appointment and arrive randomly. There are only a small number of these patients for each specialist.

A Wednesday PM session consists of three medical specialists $\left(S_{\mathrm{W} 1}\right.$ to $\left.S_{\mathrm{W} 3}\right)$ and one resident $\left(\mathrm{R}_{\mathrm{W}}\right)$ who practice for the patients referred from other departments in a hospital. Similarly a Friday morning session has five medical specialists $\left(\mathrm{S}_{\mathrm{F} 1}\right.$ to $\mathrm{S}_{\mathrm{F} 4}$ and $\left.\mathrm{S}_{\mathrm{FE}}\right)$ and one resident $\left(\mathrm{R}_{\mathrm{F}}\right)$. One specialist in a Friday morning session, denoted as $\mathrm{S}_{\mathrm{FE}}$, only sees post-surgery consultation patients.

Additional descriptions related to the simulation model construction are listed below:

1. Each patient is pre-assigned to a doctor according to a selected appointment rule,

2. A session lasts for four hours, and working time after the session is considered as overtime,

3. Arrivals of walk-in patents are approximated to follow Poisson process with the rate of $8 \mathrm{ppl} / \mathrm{hr}$,

4. A doctor leaves work when he finishes his line of patients,

5. Mydriasis is only available for those who have arrived within three hours from the beginning of a session,

6. Service time for each doctor is independent and identically distributed, regardless of the level of the congestion in the system, and

7. For each simulation experiment, the number of simulation run replication is 100 .

\section{RESULTS}

The objective of this study is to compare the effectiveness of two sets of appointment scheduling solutions for a multiple-doctor, resource-sharing outpatient department. The first set is obtained by selecting a seemingly best scheduling rule for each doctor, and simply combining those to form a set of collective scheduling rules for a session. The other set is given by the best performing set among the ones tested in the orthogonal array experiments. As there are nine combinations of appointment rules tested in the experiment - both for Wednesday session and Friday session -, we call this a best-from-the-nine set, noted as BF9.

First, the patient flow performance of each doctor under three scheduling rules is evaluated in order to find the most favorable rule for individual doctors. The cost function, $f$, specified by equation (1) is computed for each doctor $k$.

$$
f_{k}=C_{W} W^{k}+C_{O} O^{k}
$$

$f_{k}$ represent the patient flow cost function for doctor $k$, and $W^{k}$ and $O^{k}$ are the average waiting time (minute) of doctor $k$ 's patients and the overtime (minute) of doctor $k$. Then, the rule that yields the minimum value of $f_{k}$ is selected as the most preferred rule for each doctor $k$ and these rules are combined to define a set of overall scheduling rules for a session. 
Next step is to evaluate the performance of the individually selected rules against the performance of the best-from-the-nine solution identified from the orthogonal array experiments. The performance of these solutions is measured for the entire system by equation (1) as the value of $\left(C_{W}, C_{O}\right)$ changes. If the main effects of design parameters dominate, then the first set performs better than the second. Otherwise, the main effects of design parameters alone cannot explain majority of a system's performance, so an appointment scheduling of multiple-doctor and common resources requires systematic approaches.

\subsection{Wednesday PM Sessions}

Based on the experiments conducted, the most favorable scheduling rules for each doctor are found as $\left(C_{W}, C_{O}\right)$ changes. The best rule for each doctor depends upon the value of $\left(C_{W}, C_{O}\right)$. For $\mathrm{S}_{\mathrm{w} 1}$, rule IBFT dominates the other rules for all $C_{W}$ and $C_{O}$ values, whereas for $S_{\mathrm{w} 2}$ and $\mathrm{S}_{\mathrm{w} 3}$, different rules perform best depending on a specific value of the coefficients.

(a) Efficient Frontier of $S_{\mathrm{W} 1}$

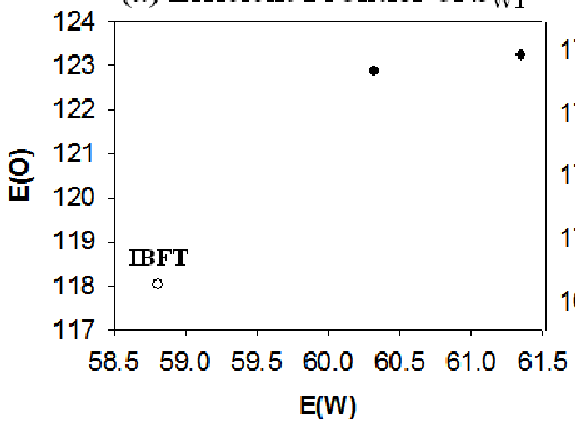

(b) Efficient Frontier of $S_{W_{2}}$

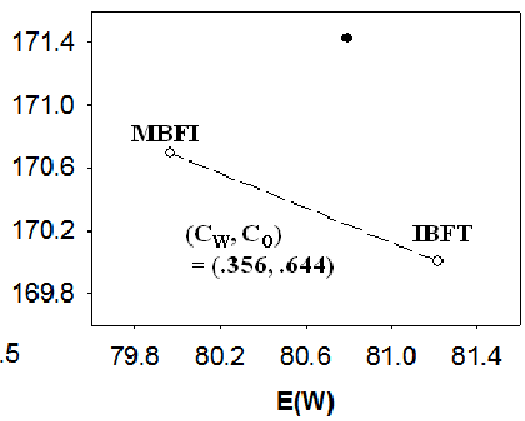

(c) Efficient Frontier of $S_{\mathrm{W} 3}$

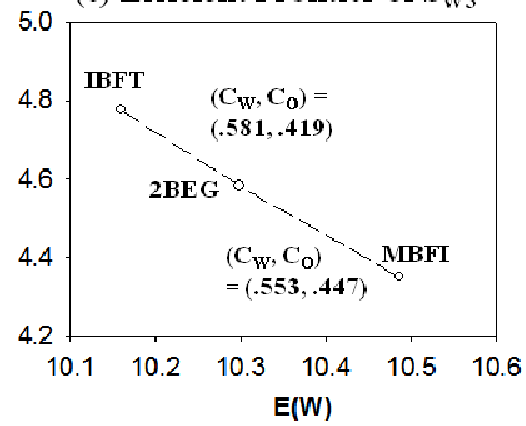

Figure 2: Efficient frontier for $\mathrm{S}_{\mathrm{W} 1}, \mathrm{~S}_{\mathrm{W}_{2}}$ and $\mathrm{S}_{\mathrm{W} 3}$. Average of overtime and waiting time are calculated based on 100 simulation replications. The unit of waiting time and overtime is minute.

With this result, we can form a set of most preferred appointment scheduling rule for the three doctors for a range of $\left(C_{W}, C_{O}\right)$. As shown in Figure 3, four different combinations of scheduling rules are identified for four intervals of $\left(C_{W}, C_{O}\right)$. Since $C_{W}$ and $C_{O}$ sum up to 1 , Figure 3 is drawn for $C_{W}$ values.

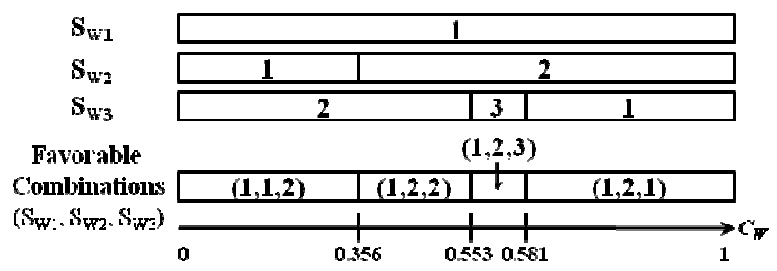

Figure 3: Combinations of the most favorable rules for the three doctors as the value of $\left(C_{W}, C_{O}\right)$ changes. Level 1, 2 and 3 refer to IBFT, MBFI and 2BEG respectively.

The result above shows that a set of the most favorable rules differs depending on the value of $\left(C_{W}\right.$, $C_{O}$ ). Similarly, it is expected that for each interval of $C_{W}$, the best-from-the-nine will be different as well. For fair comparison, BF9 that is determined for a specific value of $C_{W}$ is compared to the corresponding combinations of the most favorable rules set. For performance analysis, $C_{W}$ is chosen arbitrarily in each interval. For example, $C_{W}=0.2$ is chosen for the $C_{W}$ interval $[0,0.356]$ and the BF9, which is the best rule set out of orthogonal arrays, for this value is (MBFI, IBFT, MBFI), whereas the favorable rule set is (IBFT, IBFT, MBFI). The comparison result is shown in Figure 4. It shows that for every interval of $C_{W}$, the BF9 outperforms the corresponding set of most favored rules. This confirms the prediction that a collection of the scheduling rules that are individually favored by each doctor do not necessarily yield the 
best result for the entire system. For some doctors, a rule that is most preferred by him/her is not an optimum solution when considered in the context of the whole system.

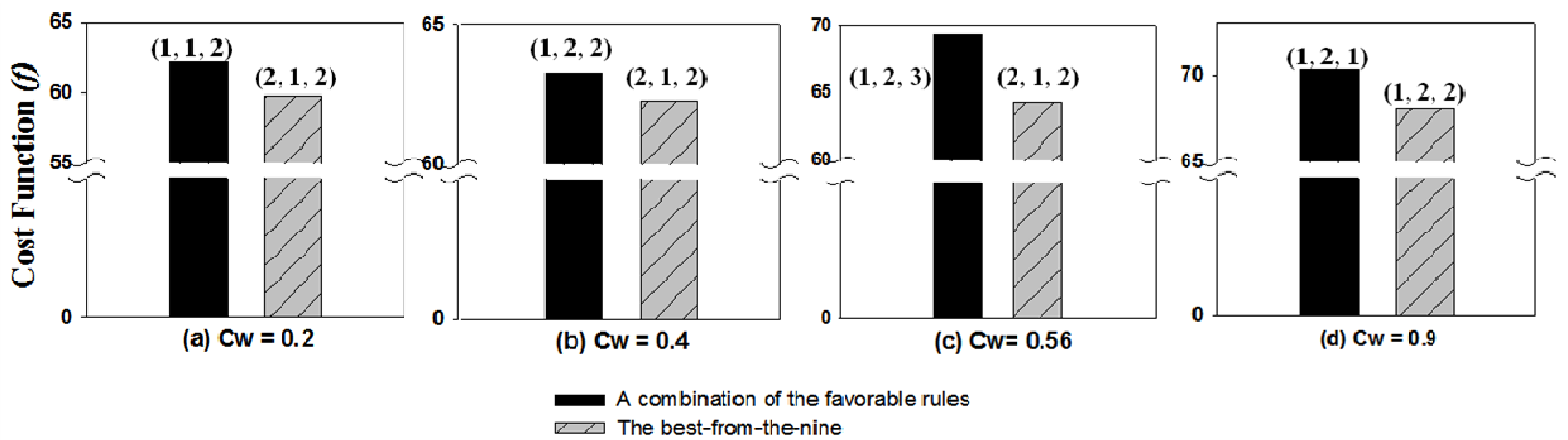

Figure 4: Comparison of the cost function between the most favorable rule set and the best-from-the-nine set for four $C_{W}$ values. The shorter cost function bar represents the better performance. The cost function was obtained from 100 run replications. The parenthesis above/next to each bar represents scheduling rules of $\left(\mathrm{S}_{\mathrm{W} 1}, \mathrm{~S}_{\mathrm{W} 2}, \mathrm{~S}_{\mathrm{W} 3}\right)$, and level 1,2 and 3 refer to IBFT, MBFI and 2BEG respectively.

\subsection{Friday AM Sessions}

Similarly, the result for Friday AM sessions is obtained. As shown in Figure 5, $\mathrm{S}_{\mathrm{F} 3}$ and $\mathrm{S}_{\mathrm{F} 4}$ have one universally favorable rule, but $S_{\mathrm{F} 1}$ and $S_{\mathrm{F} 2}$ have different solutions as value of $\left(C_{W}, C_{O}\right)$ changes. Figure 6 shows three sets of most favorable rules per three $C_{W}$ intervals.

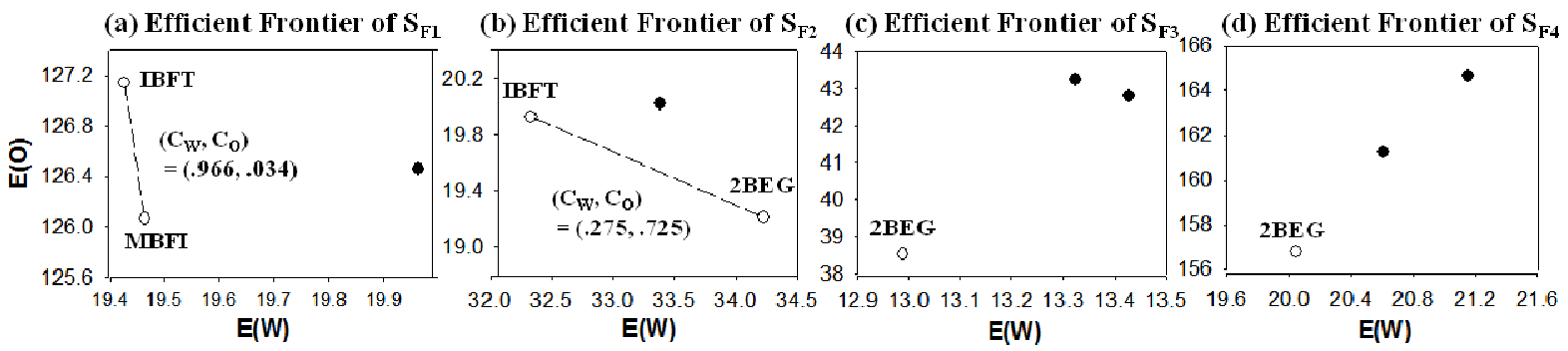

Figure 5: Efficient frontiers for $\mathrm{S}_{\mathrm{F} 1}, \mathrm{~S}_{\mathrm{F} 2}, \mathrm{~S}_{\mathrm{F} 3}$ and $\mathrm{S}_{\mathrm{F} 4}$. Average of overtime and waiting time are calculated based on 100 simulation replications.

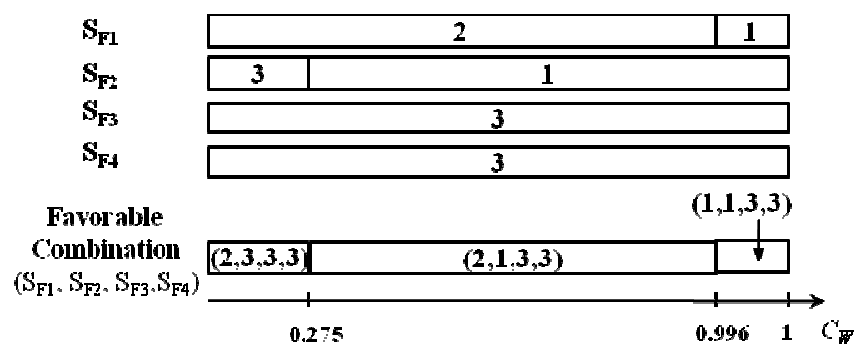

Figure 6: Combinations of the most favorable rules for the three doctors as the value of $\left(C_{W}, C_{O}\right)$ changes. Level 1, 2 and 3 refer to IBFT, MBFI and 2BEG respectively.

As shown in figure 6, Combinations of the most favorable rules for the four doctors in Friday PM Sessions are $\left(\mathrm{S}_{\mathrm{R} 1}, \mathrm{~S}_{\mathrm{F} 2}, \mathrm{~S}_{\mathrm{F} 3}, \mathrm{~S}_{\mathrm{F} 4}\right)=(\mathrm{MBFI}, 2 \mathrm{BEG}, 2 \mathrm{BEG}, 2 \mathrm{BEG})$, (MBFI, IBFT, 2BEG, 2BEG) and (IBFT, 
IBFT, 2BEG, 2BEG) for $\mathrm{C}_{\mathrm{W}}$ intervals of $[0,0.275],[0.275,0.996]$ and $[0.996,1]$ respectively. The cost function, $f$, of those sets are compared to BF9 in each interval of $C_{W}$.

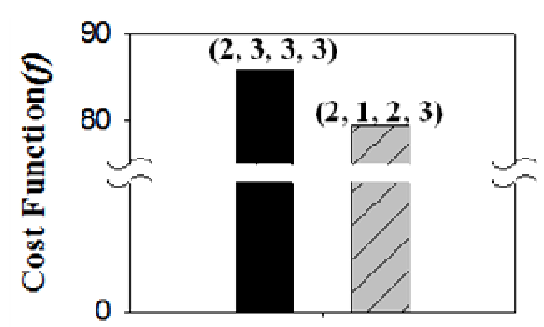

(a) $C_{w}=0.1$

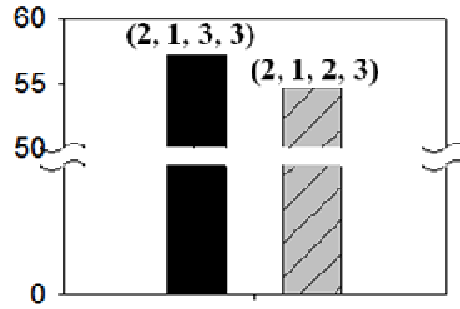

(b) $\mathrm{Cw}_{\mathrm{w}}=0.5$

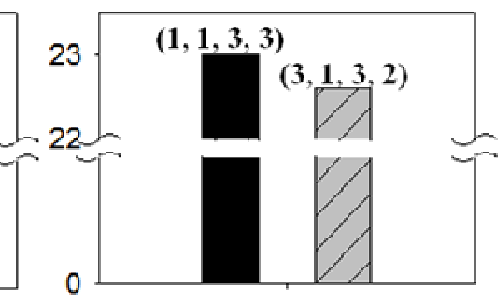

(c) $\mathrm{Cw}_{\mathrm{w}}=1$

A combination of the favorable rules

Figure 7: Comparison of the cost function between the most favorable rule set and the best-from-the-nine set for four $C_{W}$ values. The shorter cost function bar represents the better performance. The cost function was obtained from 100 run replications. The parenthesis above/next to each bar represents scheduling rules of $\left(\mathrm{S}_{\mathrm{F} 1}, \mathrm{~S}_{\mathrm{F} 2}, \mathrm{~S}_{\mathrm{F} 3}, \mathrm{~S}_{\mathrm{F} 4}\right)$, and level 1, 2 and 3 refer to IBFT, MBFI and 2BEG respectively.

Similar to Wednesday Sessions, a combination of the favorable rules does not yield the smaller value of the cost function in any of the tested values of $C_{W}$ than the best rule set obtained from orthogonal arrays. It implies that patient flows in the same session should not be assumed as independent where multiple doctors practice simultaneously and share common resources.

\section{DISCUSSION}

For an outpatient unit where multiple doctors practice together, the simplest means to deciding outpatient appointment scheduling rules is to assign one scheduling rule, which is known to perform well in general, to all doctors in a session. But it is evident that this approach would not yield better performance compared to assigning individually tailored scheduling rules to each doctor.

Then the question is on what rationale a scheduling rule should be selected for each doctor. A simpleminded answer is to find the most favorable scheduling rule to each individual doctor and assign it. This simple approach would provide the best solution when the patient flow of each doctor is independent to each other. In such case, a scheduling rule chosen by one doctor will not affect the patient flows of other doctors, and therefore the optimality is guaranteed.

When multiple doctors share resources, however, their patient flows affect each other. Our experiments clearly show that patient flows of simultaneously practicing doctors in a same session have some effects on each other. Therefore, the performance of the overall patient flows within the system is affected by this interdependency. In the presence of interdependency of patient flows, appointment scheduling problem for multi-doctor sharing resources should be considered as a system problem. An optimal set of patient appointment scheduling rules should be derived by considering all doctors and their patient flows collectively. One way is to conduct full factorial experiments to find the best scheduling set. However, when there are many doctors working in a session and candidate appointment rules considered are numerous, the problem size of finding an optimal set can be too large to perform full factorial experiments. In such cases, DOE can be used to reduce the number of experiments to conduct.

There are a few limitations in this study. Since the data for arrival times of walk-in patients was unavailable, their inter-arrival time distribution is assumed to follow exponential distribution. Although this assumption is supported by a few prior researches (Swarzman (1970), Cayirli (2006)), its validity was not tested with respect to the model used in this study.

In addition, the correlation between a morning and afternoon session in a day is not fully considered, especially for the resource pre-occupations. When there are many patients in the morning session, it is 
very possible that some of these patients are attended in the afternoon session. Since doctors working in the morning session and afternoon session are different, this overflow does not cause a correlation problem for doctor component of the system. However, for other common resources such as exam rooms and test equipments, patients from the morning session will affect the afternoon session's patient flow. Under the circumstances, shared resources are preoccupied that could result in longer waiting time and overtime. Such correlation was not taken into our consideration.

There may be practical limitations in applying this system-oriented solution. In our example, the best solution selected from the experiments places most of the cost penalty to one doctor, while waiting time and overtime is reduced for others. It means, in practice, that a few doctors in the unit and their patients experience long overtime and waiting time for the benefit of the majority. This imbalance can be perceived as unfairness, and the solution may not be acceptable in reality. This problem may be addressed by using an alternative objective function such as minimizing the variance of cost penalties across different doctors.

\section{CONCLUSION}

Many outpatient units have multiple doctors practicing simultaneously, sharing common resources. Mostly, these doctors practice independently and have their own patient panel. Decisions each doctor makes regarding his or her patient flow determine the patient flow performance of the entire unit such as patients' waiting time and staff overtime work. When doctors share common resources, individual doctor's decision affects the patient flow of other doctors.

As such, appointment scheduling under multiple-doctor, resource-sharing environment must consider this interdependency among the patient flows by multiple doctors. A mere collection of seemingly best rules for individual doctors do not necessarily lead to the overall optimal performance. It is important to recognize the existence of interdependency between patient flows, and appointment scheduling under such environment should be derived by considering the entire system as a whole.

\section{ACKNOWLEDGMENT}

The authors thank to Youngwoo Lee for data collection and preparation and simulation model construction. 


\section{A INPUT DATA SET VALUE}

Table 7: Representative examples and corresponding values of input data set are given. This table is detailed version of Table 2. (Time unit: Minute)

\begin{tabular}{|c|c|}
\hline Data Type & Representative Examples \\
\hline $\begin{array}{l}\text { Service time } \\
\text { Distribution }\end{array}$ & $\begin{array}{l}\text { Consultation time: } \\
-\quad \mathrm{S}_{\mathrm{F} 1} \text { 's consultation time: Triangle }(2.5,3,3.5) \\
-\quad \mathrm{S}_{\mathrm{F} 4} \text { 's consultation time: Triangle }(6,7,8) \\
\text { Medical Examination time: } \\
-\quad \text { Dilatation of the pupil: Triangle }(20,30,60) \\
-\quad \text { Eyesight test time of } \mathrm{S}_{\mathrm{F} 1} \text { 's patients: Triangle }(1.8,2,3) \\
-\quad \text { Eyesight test time of } \mathrm{S}_{\mathrm{F} 4} \text { s patients: } \\
\quad \text { Triangle }(1.8,2,3)+\operatorname{Triangle}(1.8,2,3)+\text { Triangle }(0.8,1,1.5) \\
\quad \text { (Sum of three different processes for } \mathrm{S}_{\mathrm{F} 4} \text { 's patients) }\end{array}$ \\
\hline $\begin{array}{l}\text { Walk-in Patients } \\
\text { Distribution }\end{array}$ & $\begin{array}{l}\text { - Inter-arrival time distribution of walk-in patients } \\
-\quad \text { Exponential } \sim(7.5)\end{array}$ \\
\hline Punctuality Distribution & - $\quad$ Normal $\sim(-25.3,54.66)$ \\
\hline No-show Ratio & - $13 \%($ Constant $)$ \\
\hline New-patient Ratio & $\begin{array}{l}\text { Newcomer ratio of doctors: } \\
-\quad \text { Newcomer ratio of } \mathrm{S}_{\mathrm{F} 1}: 16 \% \\
-\quad \text { Newcomer ratio of } \mathrm{S}_{\mathrm{F} 4}: 22 \% \\
\end{array}$ \\
\hline Process Pattern Ratio & $\begin{array}{l}\mathrm{S}_{\mathrm{F} 1} \text { 's Process Pattern Ratio: } \\
- \text { Consultation: } 44 \% \\
-\quad \text { Consultation - Examination: } 4 \% \\
-\quad \text { Consultation - Examination - Consultation: } 30 \% \\
- \text { Examination: } 22 \% \\
- \text { Examination - Consultation: } 0 \% \\
\end{array}$ \\
\hline $\begin{array}{l}\text { Post-consultation } \\
\text { Examination Ratio }\end{array}$ & $\begin{array}{l}\mathrm{S}_{\mathrm{F} 1} \text { 's Examination ratios: } \\
-\quad \text { Exam1: } 33 \% \\
-\quad \text { Exam2: } 19 \% \\
-\quad \text { Exam3: } 20 \% \\
-\quad \text { Exam4: } 9 \% \\
-\quad \text { Exam5: } 7 \% \\
-\quad \text { Exam6: } 10 \% \\
\left(\mathrm{~S}_{\mathrm{F} 1} \text { requests } 6 \text { kinds of medical examinations only) }\right.\end{array}$ \\
\hline Resource capacity & $\begin{array}{l}\text { - Personnel in Eyesight examination: } 3 \text { Persons } \\
\text { - Physician assistants in a medical interview room: } 2 \text { Persons } \\
\text { - Personnel in an Exam room: } 1 \text { Person } \\
\text { - Number of exam rooms: } 4 \text { Rooms }\end{array}$ \\
\hline
\end{tabular}

\section{B ORTHOGONAL ARRAYS}

Table 3: Experiment settings of Wednesday PM Sessions. Level 1, 2 and 3 refer to IBFT, MBFI and 2BEG respectively. The orthogonal array of 3-factor and 3-level is adopted from Minitab, statistical software. 
Yeon, Lee and Jang

\begin{tabular}{c|lllllllll}
\hline $\boldsymbol{E x p . \#}$ & $\boldsymbol{1}$ & $\mathbf{2}$ & $\mathbf{3}$ & $\mathbf{4}$ & $\mathbf{5}$ & $\mathbf{6}$ & $\mathbf{7}$ & $\boldsymbol{8}$ & $\mathbf{9}$ \\
\hline $\mathbf{S}_{\mathbf{W} 1}$ & 1 & 1 & 1 & 3 & 3 & 3 & 2 & 2 & 2 \\
$\mathbf{S}_{\mathbf{W} 2}$ & 1 & 3 & 2 & 1 & 3 & 2 & 1 & 3 & 2 \\
$\mathbf{S}_{\mathbf{W} 3}$ & 1 & 3 & 2 & 3 & 2 & 1 & 2 & 1 & 3 \\
\hline
\end{tabular}

Table 4: Experiment settings of Friday AM Sessions. Level 1, 2 and 3 refer to IBFT, MBFI and 2BEG respectively. The orthogonal array of 4-factor and 3-level is adopted from Minitab.

\begin{tabular}{c|lllllllll}
\hline Exp.\# & $\mathbf{1}$ & $\mathbf{2}$ & $\mathbf{3}$ & $\mathbf{4}$ & $\mathbf{5}$ & $\mathbf{6}$ & $\mathbf{7}$ & $\mathbf{8}$ & $\mathbf{9}$ \\
\hline $\mathbf{S}_{\mathbf{F} 1}$ & 1 & 1 & 1 & 3 & 3 & 3 & 2 & 2 & 2 \\
$\mathbf{S}_{\mathbf{F} 2}$ & 1 & 3 & 2 & 1 & 3 & 2 & 1 & 3 & 2 \\
$\mathbf{S}_{\mathbf{F 3}}$ & 1 & 3 & 2 & 3 & 2 & 3 & 2 & 1 & 3 \\
$\mathbf{S}_{\mathbf{F} 4}$ & 1 & 3 & 2 & 2 & 1 & 1 & 3 & 2 & 1 \\
\hline
\end{tabular}

\section{REFERENCES}

Bailey, N. T. J. 1952. A study of queues and appointment systems in hospital outpatient departments, with special reference to waiting-times. Journal of the Royal Statistical Society 14:185-199.

Blanco White and Pike. 1964. Appointment systems in out-patient' clinics and the effect of patients' unpunctuality, Medicare, 2, 133-145.

Cayirli, T., E. Veral, 2003. Outpatient scheduling in health care: A review of literature. Production and Operations Management 12:519-549

Cayirli, T., E. Veral, and H. Rosen. 2006. Designing appointment scheduling systems for ambulatory care services. Health Care Management Science 9:47-58

Cayirli, T., E. Veral, and H. Rosen. 2008. Assessment of patient classification in appointment system design", Production and Operations Management 17:338-353

Swarzman G. 1970. The patient arrival process in hospitals: Statistical analysis. Health service research 5: $320-329$

\section{AUTHOR BIOGRAPHIES}

NARA YEON is a Master candidate of Industrial \& Systems Engineering Department at KAIST, South Korea. Her research interest is in the area of system design and modeling and simulation especially in healthcare systems. She received her undergraduate degree in Industrial \& Systems Engineering Department at KAIST. Her email address is <narayeon@kaist.ac.kr>.

Dr. TAESIK LEE is an assistant professor of Industrial \& Systems Engineering Department at KAIST, Korea. His research interests are primarily in the area of system design, modeling and simulation, especially for large-scale, complex systems. For the past few years, he has been working on re/designing various aspects of healthcare delivery systems. Dr. Lee obtained his MS and PhD degree from MIT in the Dept. of Mechanical Engineering. His email address is <taesik. lee@kaist. edu>.

HOON JANG is a Ph.D candidate in Industrial and Systems Engineering Department at KAIST. He received his undergraduate and master degree in Industrial and Systems Engineering Department at KAIST. Korea. His research interests include simulation and modeling in healthcare delivery systems. His email is <hoon. jang@kaist.ac.kr>. 